\title{
Edad y crecimiento del híbrido de tilapia Oreochromis niloticus $x$ Oreochromis aureus (Perciformes: Cichlidae) en la represa “Zimapán” Hidalgo, México
}

\author{
M. A. Gómez-Ponce ${ }^{1}$, K. Granados-Flores ${ }^{2}$, C. Padilla $^{2}$, M. López-Hernández ${ }^{1} \&$ \\ G. Núñez-Nogueira ${ }^{1 *}$ \\ 1. Estación "El Carmen", Instituto de Ciencias del Mar y Limnología, Universidad Nacional Autónoma de México, \\ Ciudad del Carmen, Campeche, México. CP. 24157; mgomez@cmarl.unam.mx; gnunez@cmarl.unam.mx \\ 2. Unidad Académica Ecología y Biodiversidad Acuática, Instituto de Ciencias del Mar Limnología, Universidad \\ Nacional Autónoma de México, México.CP 04510; delfinkgf2105@yahoo.com.mx; padillacm@yahoo.com.mx; \\ martinlimnos@yahoo.com.mx \\ * Correspondencia.
}

Recibido 06-VII-2010. Corregido 24-X-2010. Aceptado 24-XI-2010.

\begin{abstract}
Age and growth of the hybrid tilapia Oreochromis niloticus x Oreochromis aureus (Perciformes: Cichlidae) in the dam "Zimapan" Mexico. Studies on age and growth in fishes are essential to establish models on population dynamics. The previos issues were determined in this study by scale growth analyses. Between September 2003 and May 2004, 382 organisms were captured using gill and atarraya nets. Eight samples were developed every month a long this study. Standard length in centimeters $\left(L_{e}\right)$, total weight in grams $\left(P_{t}\right)$, scale length in centimeters $\left(L_{\text {esc }}\right)$ and growth rings were determined. The size and weight ranged from 38 to $232 \mathrm{~mm}\left(\mathrm{~L}_{\mathrm{e}}\right)$ and 8.7 to $311.9 \mathrm{~g}\left(\mathrm{P}_{\mathrm{t}}\right)$, respectively. The growth rings analyses reveal four age groups, being the second group the most representative with $34.3 \%$ of the total sampled. The scale ring analyses showed two growth rings, one develops between November-January and the other between April-May, in good correlation with low temperature and gonad development, respectively. The growth parameters were established as: $\mathrm{L}_{\infty}=281.1 \mathrm{~mm}$, $\mathrm{W}_{\infty}=877.1 \mathrm{~g}, \mathrm{~K}=0.33$ and $\mathrm{T}_{0}=0.88 \mathrm{year}^{-1}$. The growth curves based on length and weight were described by using the von Bertalanffy model: $\mathrm{L}_{\mathrm{e}}=28.11\left[1-\mathrm{e}^{-0.33(t+0.88)}\right]$ and $\mathrm{P}_{\mathrm{t}}=877.17\left[1-\mathrm{e}^{-0.33(t+0.88)}\right]^{3}$. Finally, it is concluded that commercial catches in this dam are between a year and year and half-old when exploited. Rev. Biol. Trop. 59 (2): 761-770. Epub 2011 June 01.
\end{abstract}

Key words: fishery, growth rings, reproductive index, scales.

La pesca de tilapia en México se ha convertido en una de las pesquerías de agua dulce más importantes, la producción en el 2000 fue de 74 734ton (Anónimo 2001) y representa el $5.3 \%$ de la producción total nacional de captura en aguas interiores. El uso de especies con un alto índice reproductivo como la tilapia (Oreochromis spp.) se caracteriza por presentar un rápido crecimiento y una fácil comercialización, debido a que son considerados como productos relativamente baratos y de fácil adquisición (Díaz 1985, Gómez-Márquez et al. 2008). Esta situación ha mejorado los estándares de vida de las comunidades rurales relacionadas con la pesquería (Beltrán -Álvarez et al. 2009).

La determinación de la edad de cualquier organismo, es de gran interés, ya que permite realizar estudios sobre crecimiento; conocer la longevidad de las especies, la producción de biomasa y los niveles de captura para cada edad; identificar las clases anuales que componen a la población; determinar la edad de primera madurez y de reclutamiento, además de calcular la tasa de mortalidad a la cual está sujeta la población. 
Los métodos para determinar la edad de los peces pueden dividirse en directos e indirectos. Los primeros están basados en la interpretación de capas depositadas en las partes calcáreas del pez, las cuales están relacionadas con la observación directa de marcas de crecimiento. Éstas son el producto de cambios en el metabolismo de los individuos y están correlacionadas con el tiempo en que ocurrieron (Ehrhardt 1981). Ahora bien, los segundos que son los métodos indirectos se basan en el análisis de frecuencias de tallas de una población de peces, éstos normalmente ayudan a confirmar otros procedimientos, particularmente aquellos que involucran la interpretación de la edad a partir de estructuras calcificadas (Pauly 1983, Weatherley \& Gill 1987, Sparre \& Venema 1997). La interpretación de la edad y crecimiento a partir de estructuras duras se basa en el supuesto de que rasgos periódicos son formados de manera constante y que la distancia entre rasgos consecutivos, tales como los anillos en escamas, son proporcionales al crecimiento del pez, por ello el crecimiento de las estructuras puede reflejar la talla del pez (Campana \& Neilson 1985).

Asimismo, los cambios en el metabolismo durante ciertos periodos se ven reflejados como capas en las partes duras, las cuales son causadas principalmente por cambios estacionales en alimentación, temperatura y reproducción (Gómez-Márquez 1998, Gómez-Márquez et al. 2008).

Asimismo, se ha observado que estos cambios metabólicos en los peces origina una banda de crecimiento ancha en condiciones óptimas, mientras que en situaciones de estrés motivan un escaso aumento de dicha banda (Granado 1996). Generalmente, el crecimiento es rápido durante los meses calurosos y se vuelve lento en los meses de invierno (Schneider et al. 2000).

Los cíclidos se distribuyen predominantemente en climas tropicales, donde las estaciones anuales no son muy marcadas, y por consecuencia el crecimiento de las especies tiende a ser discontinuo, ésto se convierte en una desventaja cuando se aplican métodos en análisis de frecuencias de tallas para la determinación de la edad. Mientras, que las estructuras óseas han resultado ser muy útiles si se logra definir claramente su periodicidad (Weatherley \& Gill 1987, Morales-Nin 1989).

Pese a que en la represa Zimapán las capturas han sobrepasado las 1000 toneladas en los últimos años, los estudios biológicos y pesqueros en este embalse son escasos. Ante esta falta de información, el objetivo del presente estudio fue determinar la edad y describir el crecimiento de las tilapias en el embalse.

\section{MATERIALES Y MÉTODOS}

Área de estudio: Zimapán $\left(20^{\circ} 35^{\prime}\right.$ $20^{\circ} 40^{\prime} \mathrm{N}$ y $99^{\circ} 22^{\prime}-99^{\circ} 37^{\prime}$ ) es una represa con una superficie media de 2600 ha de espejo de agua, situada a una altitud de $1870 \mathrm{~m}$ sobre el nivel del mar y alimentada principalmente por los Ríos San Juan y Tula. En la parte occidental del Estado de Hidalgo colinda con los municipios de Zimapán, Tasquillo y Tecozautla y con el Estado de Querétaro colinda con el municipio de Cadereyta de Montes (Fig. 1).

Entonces, se llevaron a cabo ocho muestreos biológicos y ocho muestreos comerciales comprendidos entre septiembre del 2003 y mayo del 2004, los cuales abarcaron los principales periodos reproductivos de la tilapia (Hernández-Montaño \& Orbe 2002). Con la finalidad de garantizar la representatividad del espectro de tallas, se realizaron muestreos en diferentes localidades de la represa (Fig. 1). Los lances se llevaron a cabo con una red tipo atarraya de $1.52 \mathrm{~m}$ de radio y $0.9 \mathrm{~cm}$ de abertura de luz de malla y posteriormente, con redes agalleras con una longitud máxima de $60 \mathrm{~m}$, una caída o altura máxima de $5 \mathrm{~m}$ y una abertura de malla de $11.43 \mathrm{~cm}$.

Los organismos se identificaron mediante el uso de claves dicotómicas (Trewavas 1983, Arredondo-Figueroa \& Guzmán-Arroyo 1986), los caracteres diagnósticos que se consideraron para la identificación fueron las características del hueso faríngeo, forma de los dientes y tipo de bandeo presente en la aleta caudal (Arredondo-Figueroa \& Tejeda-Salinas 1989). 


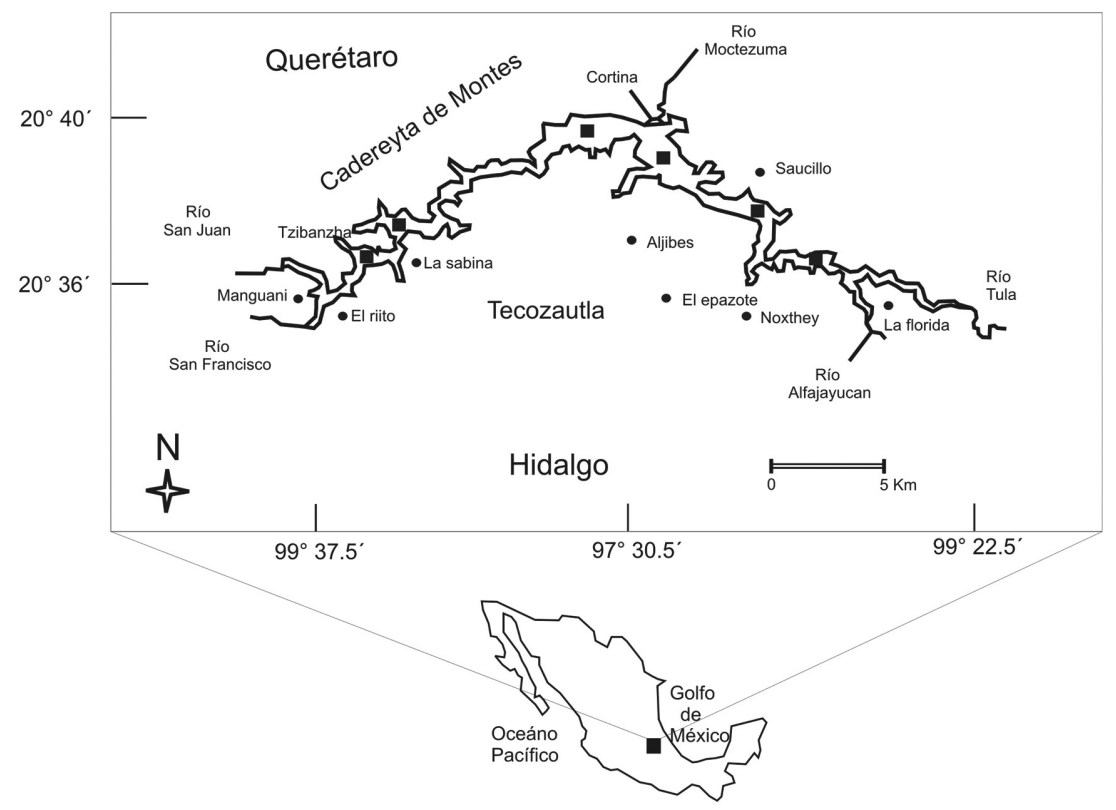

Fig. 1. Área de estudio y puntos de muestreo $(\bullet)$.

Fig. 1. Study area and sampling points $(\bullet)$.

Por lo tanto, se capturó un total de 382 organismos de los cuales se registró la longitud estándar $\left(\mathrm{L}_{\mathrm{e}}\right)$ que comprendió desde la punta de la cabeza al pedúnculo caudal, y se utilizó un ictiómetro convencional con divisiones de $1 \mathrm{~mm}$, mientras que el peso total $\left(\mathrm{P}_{\mathrm{t}}\right)$ expresado en $\mathrm{g}$, se registró con una balanza granataria de $5000 \mathrm{~g}$ de capacidad y $1 \mathrm{~g}$ de precisión.

\section{Determinación de la edad}

a. Relaciones biométricas: Para llevar a cabo el retrocálculo de las longitudes pretéritas o pasadas fue indispensable conocer saber la relación entre el crecimiento de la escama $\left(\mathrm{L}_{\text {esc }}\right)$ respecto a la longitud del pez $\left(\mathrm{L}_{\mathrm{e}}\right)$, ya que esto permite conocer la historia del crecimiento individual de los peces y la existencia de una proporcionalidad entre el crecimiento somático y el crecimiento de la estructura dura del organismo (Hare \& Cowen 1995, Meekan et al. 1998, Sirios et al. 1998). Por lo cual se evaluaron las relaciones biométricas entre el largo de la escama $\left(\mathrm{L}_{\text {esc }}\right)$ vs longitud estándar $\left(\mathrm{L}_{\mathrm{e}}\right)$ y la relación talla-peso con base en regresiones simples (Zar 1974).

b. Lectura de escamas: La edad se determinó mediante el conteo de marcas de crecimiento en las escamas obtenidas debajo de la aleta pectoral y sobre la línea lateral, éstas fueron etiquetadas en bolsas de plástico con una relación del número de organismo. En el laboratorio las escamas fueron lavadas con una solución jabonosa para eliminar el exceso de grasa y posteriormente se seleccionaron las tres mejores y se montaron entre dos porta objetos (FAO 1982). La lectura de escamas se realizó con un proyector para escamas marca Baush \& Lomb mediante luz transmitida con un objetivo de 3.2X. Al realizar la lectura de las escamas se registraron las siguientes medidas: número de anillos o marcas de crecimiento, distancia del foco hasta el radio de cada anillo de crecimiento, distancia del foco al borde de la escama, largo de la escama $\left(\mathrm{L}_{\mathrm{esc}}\right)$ y ancho de 
la escama $\left(\mathrm{A}_{\mathrm{esc}}\right)$ (Fig. 2). Entonces se registró el valor de amplificación de la imagen, con el fin de calcular la distancia real entre cada anillo de crecimiento.

\section{c. Validación de anillos de crecimiento:}

Para determinar la temporalidad de la formación de los anillos de crecimiento se realizaron los gráficos de los valores mensuales promedio de incremento marginal con sus respectivas desviaciones estándar (Bagenal \&Tesch 1978, Jearld 1983).

\section{d.- Curvas de crecimiento:}

A partir de la longitud promedio calculada a cada marca de crecimiento se construyó la clave-edad longitud, con base en la cual se obtuvieron los parámetros $\mathrm{k}, \mathrm{L}_{\infty}$, por medio del método de Ford-Waldford 1946 y la $\mathrm{t}_{0}$ se obtuvo mediante el método de Beverton \& Holt 1957. Finalmente, para describir el crecimiento en longitud y peso se utilizaron las ecuaciones de von Bertalanffy 1938.

\section{RESULTADOS}

Estructura de la población por talla $\mathbf{y}$ peso: El análisis de 383 organismos de Oreochromis aureus $x$ Oreochromis niloticus mostró que las tallas y pesos variaron de 38 a $232 \mathrm{~mm}\left(\mathrm{~L}_{\mathrm{e}}\right)$ y de 8.7 a $311.9 \mathrm{~g}\left(\mathrm{P}_{\mathrm{t}}\right)$, respectivamente.
Relación entre la longitud $\mathbf{L}_{e}$ vs $\mathbf{P}_{\mathbf{t}}$ : El modelo que se ajusto mejor a los datos fue de tipo potencial, el cual se expresó como $\mathrm{P}_{\mathrm{t}}=0.0424\left(\mathrm{~L}_{\mathrm{e}}\right)^{2.98}$ con una correlación entre $\mathrm{L}_{\mathrm{e}}$ y $\mathrm{P}_{\mathrm{t}} 97 \%$ p $<0.001$ (Fig. 3). La prueba de " $t$ " de student indicó que el valor del exponente $\mathrm{b}=2.98$ no fue significativamente diferente al valor de $3(\mathrm{t}=6.314, \mathrm{p}>0.05)$, por lo que el crecimiento de la especie de este embalse se consideró como de tipo isométrico.

Relación longitud de la escama $\left(\mathrm{L}_{\text {esc }}\right)$ y la longitud $\left(\mathbf{L}_{\mathrm{e}}\right)$ : La relación fue de tipo lineal en el cual el índice de correlación obtenido fue 0.95 con una $\mathrm{p}<0.05$, el valor del índice alométrico fue de 1.04 , lo que indicó un crecimiento de tipo isométrico entre la $\mathrm{L}_{\mathrm{esc}}$ y la $\mathrm{L}_{\mathrm{e}}$. Éste resultado apoya el uso de la longitud de las escamas como una estructura adecuada para la determinación de la edad de la tilapia (Fig. 4).

Descripción de las escamas: Las escamas de $O$. niloticus x $O$. aureus fueron clasificadas como de tipo ctenoide, por su forma rectangular y su borde superior dentado. El foco se presentó bien definido ligeramente debajo del centro de la escama y en la parte posterior se observaron ctenii, pequeñas placas alargadas o dentículos pronunciados que determinan el carácter pectinado en las escamas ctenoides

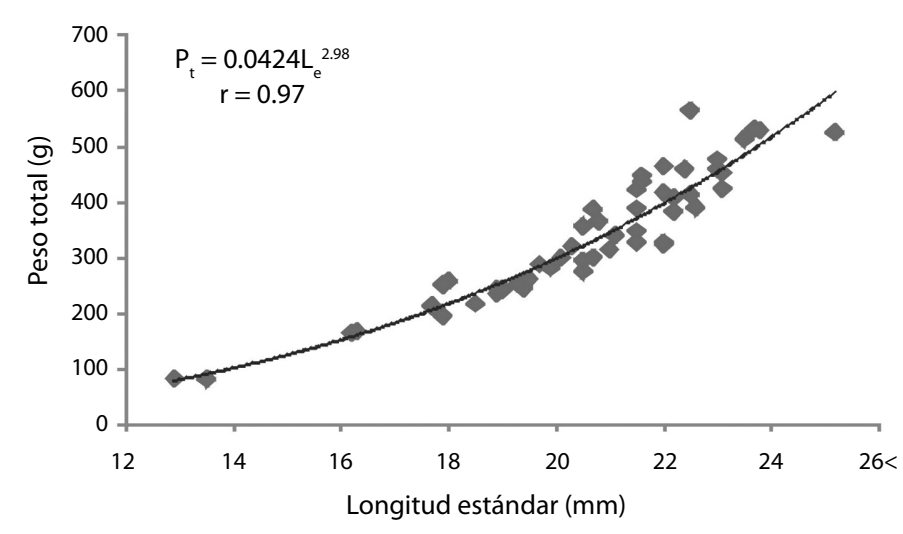

Fig. 2. Escama de la tilapia (Oreochromis niloticus $x$ Orechromis aureus).

Fig. 2. Scale of tilapia. 


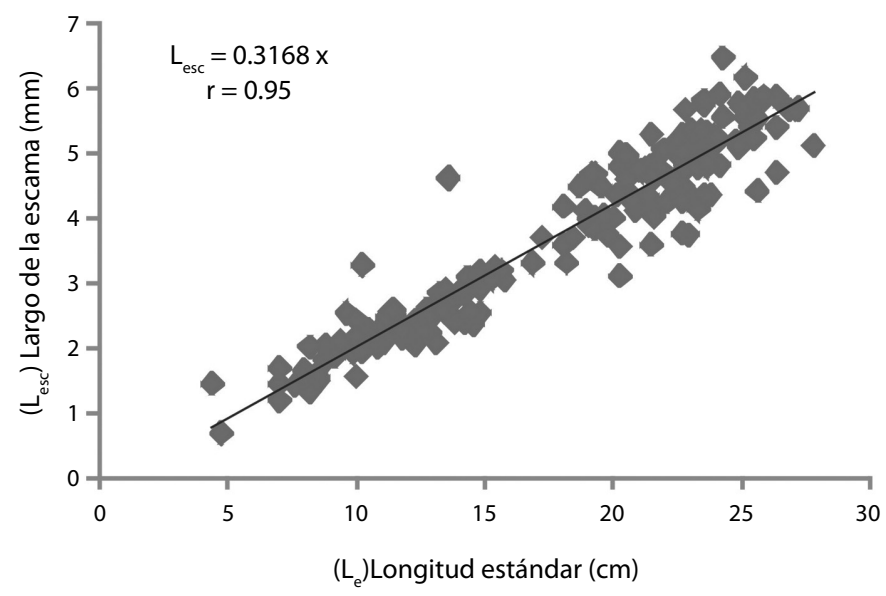

Fig. 3. Relación talla-peso de la tilapia (Oreochromis niloticus x Orechromis aureus).

Fig. 3. Length-weight relationship of Tilapia (Oreochromis niloticus x Orechromis aureus).

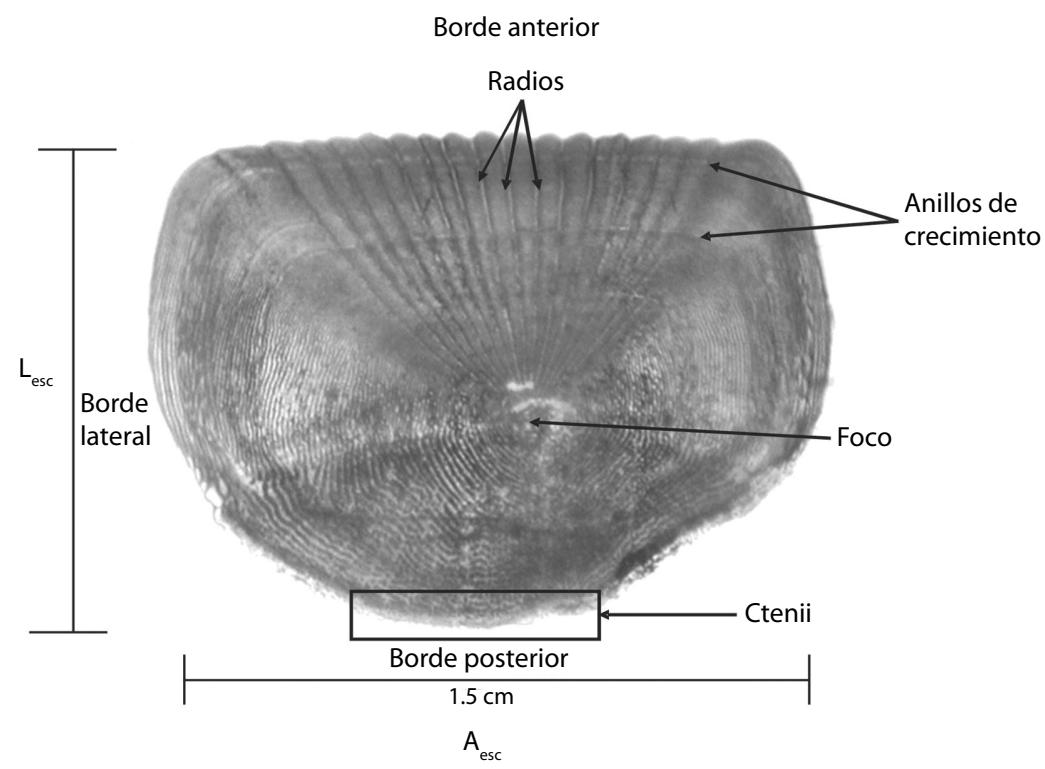

Fig. 4. Relación de la longitud estándar y la longitud de la escama de tilapia.

Fig. 4. Relationship between standard length and length of the scale of tilapia.

(Gómez-Márquez 1998). Los ctenii se encontraron en la parte expuesta de la escama y se observaron mejor en escamas de peces muy pequeños. Por lo tanto presentaron radios que convergían a partir del foco de la escama y se prolongaban hacia la parte anterior formando pequeños lóbulos. El número de radios varió de 5 a 15, sin embargo, se presentaron con mayor frecuencia escamas con 9 y 10 radios. Los anillos de crecimiento fueron identificados como una línea continua y oscura delimitada por espacios translúcidos. 
Tiempo de formación de los anillos de crecimiento: El análisis del incremento marginal mensual mostró que durante los meses de octubre y febrero se registraron los valores promedio más altos de incremento marginal, mientras que para los meses de diciembre a enero, abril y mayo se registraron los valores promedio mínimos de incremento marginal (Fig. 5).

Determinación del número de anillos de crecimiento: La lectura de escamas permitió observar de 1 a 3 anillos de crecimiento bien formados. Del total de organismos recolectados, el $33.3 \%$ presentó un sólo anillo de crecimiento el $34.3 \%$ presentó dos anillos, el $18.2 \%$ presentaron tres, finalmente el $14.2 \%$ no presentó formación.

Con base en la lectura de escamas se pudieron determinar 4 grupos de edad, cuyos valores de longitud promedio correspondieron a $7.8 \mathrm{~cm}$ para el grupo 1 (no presentaba anillo de crecimiento), $11.32 \mathrm{~cm}$ para el grupo 2 (con un solo anillo de crecimiento), $18.46 \mathrm{~cm}$ para el grupo 3 (con dos anillos de crecimiento) y finalmente $20.1 \mathrm{~cm}$ el grupo 4 (con tres anillos de crecimiento). De esta manera, se pudo construir una clave de edad longitud y obtener los parámetros de $\mathrm{K}=0.33$ /año, $\mathrm{L}_{\infty}=28.11$ /año y $\mathrm{t}_{0}=0.88 / a$ ño. Con base en estos valores se elaboraron las curva crecimiento en longitud $\mathrm{L}_{\mathrm{t}}=28.11\left(1-\mathrm{e}^{-0.33(\mathrm{t}+0.88)}\right)$ y peso $\mathrm{P}_{\mathrm{t}}=877.17\left(1-\mathrm{e}^{-}\right.$ $0.33(\mathrm{t}+0.88))^{3}$ mediante el modelo de von Bertalanffy (Fig. 6 y 7).

\section{DISCUSION}

El análisis de la relación talla $\left(\mathrm{L}_{\mathrm{e}}\right)$ longitud $\left(\mathrm{P}_{\mathrm{t}}\right)$, mostró un crecimiento de tipo isométrico en el cual los organismos presentan un crecimiento proporcional entre la talla y peso. Este mismo tipo de crecimiento ha sido reportado por Flores (2006) y Beltrán-Álvarez et al. (1997). Estudios realizados en distintas especies de tilapias y represas de México muestran que los valores de la pendiente de esta relación oscila entre 2.5 y 3.5 (Granado 1996). Esto indica que los valores obtenidos para las tilapias del embalse (2.98) se encuentra dentro de este intervalo, y da confiabilidad a los resultados obtenidos en el presente estudio.

El incremento marginal para las tilapias de Zimapán mostró dos periodos de formación de los anillos de crecimiento, el primero durante los meses fríos de noviembre a enero, debido quizás a una reducción significativa del metabolismo basal como consecuencia de las bajas temperaturas que se presentan en la represa. Y un segundo periodo de formación,

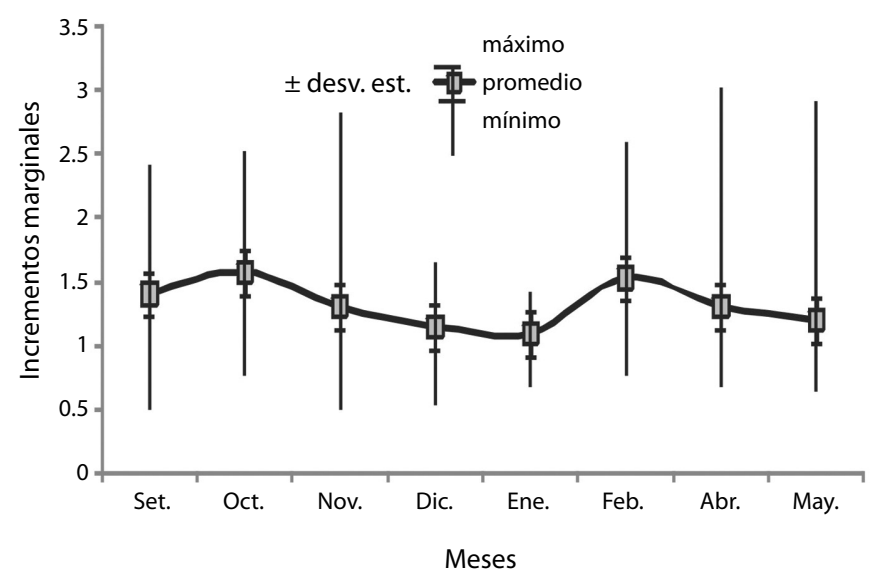

Fig. 5. Variación del incremento marginal mensual.

Fig. 5. Variation of monthly marginal increment. 


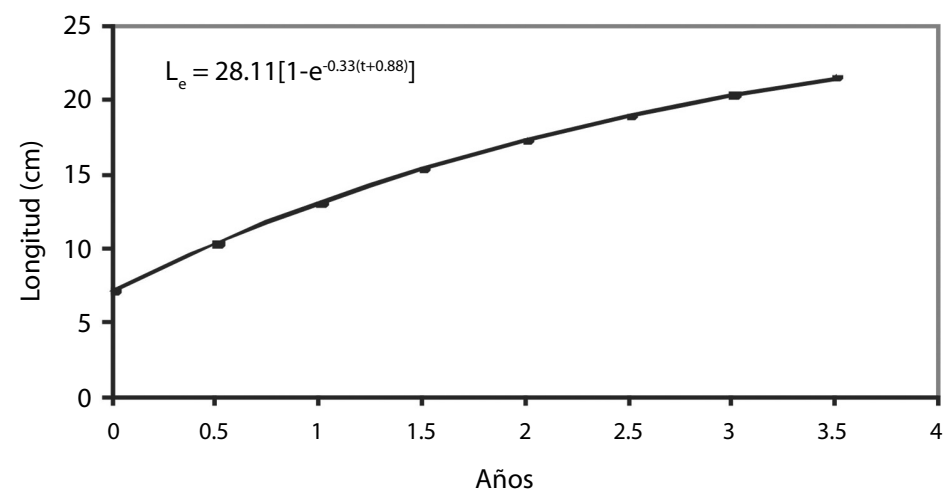

Fig. 6. Curva de crecimiento en longitud con base en el modelo de von Bertalanffy.

Fig. 6. Length growth curve based on the von Bertalanffy model.

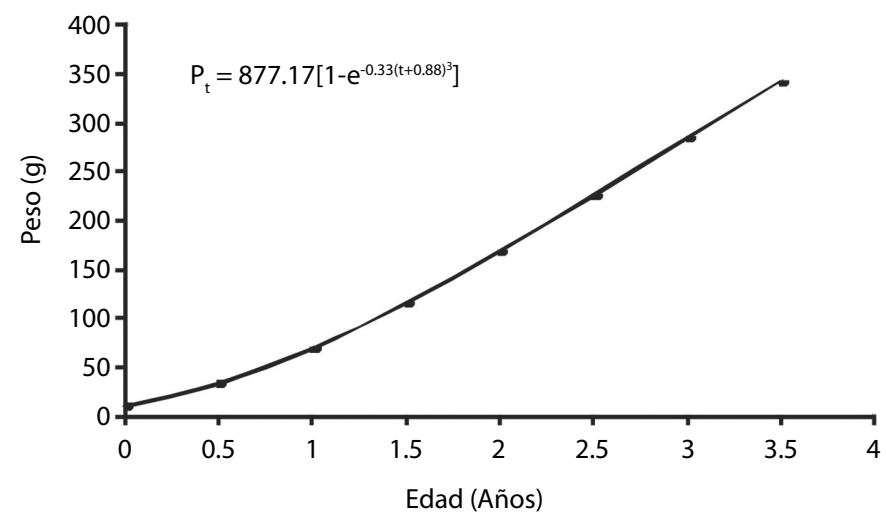

Fig. 7. Curva de crecimiento en peso con base en el modelo de von Bertalanffy.

Fig. 7. Weight growth curve based on the von Bertalanffy model.

durante los meses de abril-mayo que pudiera estar relacionado con la utilización de energía para la maduración gonádica por un lado, y por el otro, debido a que durante la incubación bucal y crianza de los alevines las tilapias no se alimentan presentando así una reducción de la tasa de crecimiento. Entonces, resultados similares fueron reportados por Gómez-Márquez (1998) quién menciona dos periodos de formación de anillos de crecimiento para la tilapia $O$. niloticus en la Laguna de Coatetelco Morelos, Gómez-Márquez atribuyó un periodo de formación de los anillos de crecimiento, durante las bajas temperaturas que prevalecen en la laguna durante la época de noviembre a enero, mientras que el otro periodo lo registró en los meses de mayo-junio, y argumenta que la interrupción del crecimiento es debido a la maduración de las gónadas y la actividad reproductiva. Jiménez-Badillo en 1999 reportó dos periodos principales de deposición de los anillos de crecimiento, uno en julio y otro en octubre, y afirmó que la formación de las marcas coincide con las principales épocas reproductivas. A su vez, Pérez \& Patlani (2002), encontraron dos periodos de deposición de anillos de 
crecimiento, en escamas de $O$. niloticus de la Represa Emiliano Zapata, ellos infirieron que la formación de anillos es de cada seis meses y el proceso de reproducción, parece ser la causa principal de la formación de los anillos como consecuencia del cambio metabólico que experimentan los peces durante la etapa reproductiva. Gómez-Márquez et al. (2008), estudiaron la edad y crecimiento de $O$. niloticus a partir del hueso opercular, en la laguna de Coatetelco, Morelos, México, y concluyeron que la formación de marcas se debía a la alta temperatura que registra el agua del embalse, a la época de estiaje y al periodo reproductivo.

Mediante el análisis de escamas, se detectaron hasta tres anillos de crecimiento, por lo que se determinaron cuatro grupos de edad para tilapia de la Represa Zimapán (Edad 1, 2, 3 y 4), la aparición de los anillos en las escamas se observó, dos veces al año, lo que indicó que la mayor captura comercial de tilapias en la represa Zimapan, se concentra en organismos que tienen entre un año y año y medio de edad. Cabe señalar que en la captura se registraron organismos de menos de un año pero en un bajo porcentaje. Es importante mencionar que los organismos del género Oreochromis pueden alcanzar edades considerables principalmente en regiones de altas latitudes, sin embargo, en algunas represas Mexicanas se tienen registros de organismos que desarrollan hasta 11 años de edad como O. aureus en la Represa Infiernillo en Michoacán (Jiménez-Badillo 2006) y $O$. niloticus en la Laguna de Coatetelco, Morelos (Gómez-Márquez 1998). En el caso de la Represa Zimapán estos organismos no alcanzan a desarrollar esas edades debido a que son capturados antes como consecuencia de la selectividad de las redes utilizadas en la captura comercial.

Estudios realizados en otros embalses y con organismos de las mismas especies se reporta valores de 56.83 para $\mathrm{L}_{\infty}, 1964.21 \mathrm{~g}$ para $\mathrm{W}_{\infty}$ y 0.13 para $\mathrm{k}$ en hembras y para machos una $\mathrm{L}_{\infty}$ de $49.25 \mathrm{~cm}$, una $\mathrm{W}_{\infty}$ de 1 $496.18 \mathrm{~g}$ y k=0.17 (Palacios 1995), cabe señalar que a pesar de que en ese estudio también se detectaron cuatro grupos de edad, las longitudes máximas fueron mayores a las del presente trabajo, sin embargo, posiblemente la $\mathrm{L}_{\infty} \mathrm{y}$ el $\mathrm{W}_{\infty}$ fueron sobreestimados debido a que el número de grupos de edad fue bajo. JiménezBadillo (1999) estimó para la población una $\mathrm{W}_{\infty}=3446 \mathrm{~g}, \mathrm{~L}_{\infty}=47.8 \mathrm{~cm}$ y $\mathrm{k}=0.46$, no obstante, determinó 11 grupos de edad mediante opérculos y escamas. Por su parte, Pérez \& Patlani (2002) registraron para hembras una $\mathrm{L}=21.15 \mathrm{~cm}, \mathrm{~W}=156.89 \mathrm{~g}$ y para machos una $\mathrm{L}_{\infty}^{\infty}=56.56 \mathrm{~cm}, \mathrm{~W}_{\infty}^{\infty}=2972.54 \mathrm{~g}$ y una $\mathrm{k}=0.13$, en la variación de estos valores en comparación con los del presente estudio, también influyen las longitudes máximas registradas por dichos autores y el reconocimiento de más grupos de edad, aunque los valores infinitos de longitud para el caso de las hembras, son más parecidos a los que se obtuvieron para las tilapias de la Represa Zimapán. Es importante remarcar que hay una variación amplia de los valores obtenidos de $\mathrm{L}_{\infty} \mathrm{y} \mathrm{W}_{\infty}$ entre ambos sexos. Aunque, es visto que la variación de los parámetros de crecimiento se debe a la amplitud de los intervalos de clases de talla, al número de clases de tallas y a la confiabilidad del muestreo. En el presente estudio se obtuvo una $\mathrm{k}$ de 0.30 anual indicativa de un rápido crecimiento para alcanzar en un menor tiempo la edad reproductiva por un lado y por el otro como un mecanismo de respuesta a la alta tasa de depredación que presentan las tilapias en la Represa Zimapán por parte de la Lobina (Micropterus salmoides). Por otra parte, se obtuvo una $\mathrm{L}_{\infty}$ de $28.1 \mathrm{~cm}$ que difiere con otros estudios previos en otros embalses, sin embargo, esto es debido a que durante el muestreo no se recolectaron organismos de tallas mayores.

\section{AGRADECIMIENTOS}

Al Gobierno del estado de Hidalgo, a las sociedades cooperativas de la Represa Zimapán. A Hernán Álvarez Guillén, Andrés Reda Deara y María Esther Diupotex Chong, por los comentarios y sugerencias para el mejoramiento del presente manuscrito. 


\section{RESUMEN}

La edad y crecimiento de la tilapia Oreochromis niloticus $x$ Oreochromis aureus fueron determinados por medio de la lectura de escamas. Un total de 382 individuos fueron capturados mediante el uso de redes agalleras y atarrayas. Ocho recolectas mensuales se llevaron a cabo entre septiembre 2003-mayo 2004; se registraron los datos de longitud estándar en $\mathrm{cm}$. $\left(\mathrm{L}_{\mathrm{e}}\right)$, el peso total en $\mathrm{g}\left(\mathrm{P}_{\mathrm{t}}\right)$ y se obtuvieron muestras de escamas para registrar el largo de la escama en $\mathrm{mm}$. $\left(\mathrm{L}_{\mathrm{esc}}\right)$ y el número de anillos de crecimiento formados. Las tallas y pesos de recolecta variaron entre 38 a $232 \mathrm{~mm}\left(\mathrm{~L}_{\mathrm{e}}\right)$ y de 8.7 a $311.9 \mathrm{~g}$ de peso total $\left(\mathrm{P}_{\mathrm{t}}\right)$ respectivamente. El recuento de los anillos anuales en escamas, permitió establecer cuatro grupos de edad, siendo el grupo de edad 2 el más representativo, con un $34.3 \%$ de la población muestreada. El cálculo de los incrementos marginales del crecimiento mensual de las escamas, sugirió la formación de dos anillos, uno de ellos se forma en los meses de noviembre-enero y otro en los meses de abril-mayo, los cuales coinciden con la época de bajas temperaturas y de madurez gonádica respectivamente. Los parámetros de crecimiento quedaron establecidos en: $\mathrm{L}_{\infty}=281.1 \mathrm{~mm}$, $\mathrm{W}_{\infty}=877.1 \mathrm{~g}, \mathrm{~K}=0.33$ y $\mathrm{T}_{0}=0.88 / \mathrm{año}$. Las curvas de crecimiento en longitud y peso se describieron mediante las ecuaciones de von Bertalanffy: $\mathrm{L}_{\mathrm{e}}=28.11\left[1-\mathrm{e}^{-0.33(\mathrm{t}+0.88)}\right]$ y $\mathrm{P}_{\mathrm{t}}=877.17\left[1-\mathrm{e}^{-0.33(\mathrm{t}+0.88)}\right]^{3}$. Finalmente, se concluyó que los peces que se explotan en la represa mediante captura comercial presentan entre un año y año y medio de edad.

Palabras clave: pesquería, anillos de crecimiento, índice reproductivo, escamas.

\section{REFERENCIAS}

Anónimo. 2001. Anuario estadístico de Pesca. Dirección General de Informática, Estadística y Documentación. Secretaría de Pesca, México D.F., México.

Arredondo-Figueroa, J.L. \& M. Guzmán-Arroyo. 1986. Actual situación taxonómica de las especies de la Tribu Tilapiini (Pises: Cichlidae) introducidas en México. An. Inst. Biol. UNAM. Ser. Zool. 56: 555-572.

Arredondo-Figueroa, J.L. \& M. Tejeda-Salinas. 1989. El hueso Faríngeo, una estructura útil para la identificación de especies de la tribu Tilapini (Pisces: Cichlidae) introducida en México. An. Inst. Biol. UNAM. Ser. Zool. 16: 58-68.

Bagenal, T.B. \& F.W. Tesch. 1978. Age and growth, p. 101136. In T.B. Bagenal (ed.). Methods for assessment of fish production in fresh water. Blackwell, Oxford, Inglaterra.
Beltrán-Álvarez, R., P.J. Sánchez, L.J.P. Ramírez, B.G. Arroyo \& E.H. Galaviz. 1997. Informe final del proyecto: estudio para evaluar las condiciones hidrobiológicas y el estado actual de la pesquería en la Presa Gustavo Díaz Ordaz "Bacurato". UAS-SEP, Sinaloa, México.

Beltrán-Álvarez, R., P.J. Sánchez, G.L. Valdez \& A.O. Salas. 2009. Edad y crecimiento de la mojarra Oreochromis aureus (Pisces: Cichlidae) en la Presa Sanalona, Sinaloa, México. Rev. Biol. Trop. 58: 325-338.

Beverton, R.J.H. \& S.J. Holt. 1957. On the dynamics of exploited fish populations. In Erzini et al. 1989. Microcomputer Application in Fisheries. Instituto Técnologico y de Estudios Superiores de Monterrey Campus Guaymas Sonora, México.

Campana, S.E. \& J.D. Neilson. 1985. Microstructure of fish otoliths. Can. J. Fish. Aquat. Sci. 42: 1014-1032.

Carlander, K.D. 1982. Standard intercepts for calculating lengths from scale measurements for some centrarchid and percid fishes. Trans. Amer. Fish. Soc. 111: 332-336.

Casselman, J.M. 1983. Age and growth assessment of fishes from their calcified structures-techniques and tools. U.S. Dep. Commer., NOAA. Tech. Rep. NMFS 8:1-17.

Díaz, L.M. 1985. Contribución al conocimiento de edad y crecimiento de Sarotherodon aureus (mojarra) de la presa "El bosque" la Encarnación, Michoacán. Tesis de licenciatura, Universidad Nacional Autónoma de México, México.

Ehrhardt, N.M. 1981. Curso sobre métodos en dinámica de poblaciones. Primera parte: estimación de parámetros poblacionales. FAO-INP, México.

FAO. 1982. Métodos de recolección y análisis de datos de talla y edad para la evaluación de poblaciones de peces. FAO, Roma, Italia.

Flores, C. 2006. Análisis de los efectos de la selectividad de las redes agalleras sobre algunos aspectos de la pesquería comercial (Oreochromis aureus), en la presa Aurelio Benassini Vizcaíno "El Salto", Sinaloa, México. Tesis de Licenciatura, Universidad Autónoma de Sinaloa, Mazatlán, Sinaloa, México.

Ford-Walford, L.A. 1946. A new graphical method of describing the growth of animal. Bull. Fish. Res. Board. Can. 191: 382. 
Gómez-Márquez, J.L. 1998. Age and growth of Oreochromis niloticus (Perciformes: Cichlidae) in Mexico. Rev. Biol. Trop. 46: 929-936.

Gómez-Márquez, J.L., B. Peña-Mendoza, H.I. SalgadoHugarte \& J.L. Arredondo-Figueroa. 2008. Age and growth of tilapia, Oreochromis niloticus (Perciformes: Cichlidae) from a tropical Shallow lake in Mexico. Rev. Biol. Trop. 56: 576-884.

Granado, L.C. 1996. Ecología de peces. Universidad de Sevilla, Sevilla, España.

Hare, J.A. \& R.K. Cowen. 1995. Effect of age, growth rate and ontogeny on otolith sizefish size relationship in bluefish, Pomatomus saltatrix, and the implications for backcalculation of size in fish early life history stages. Can. J. Fish. Aquat. Sci 52: 1909-1922.

Hernández-Montaño, D. \& A. Orbe. 2002. Documento Técnico. "Evaluación pesquera de la presa Fernando Hiriart Balderrama (Zimapán) Hidalgo-Querétaro, México.” Centro Regional de Investigación Pesquera. Patzcuaro. Instituto Nacional de la Pesca, México D.F., México.

Jearld Jr., A. 1983. Age determination, p. 301-324. In L.A. Nielsen \& D.L. Johnson (eds.). Fisheries techniques. Am. Fish. Soc. Bethesda, Maryland. Universidad Nacional Autónoma de México, México D.F., México.

Jiménez-Badillo, L. 1999. Análisis de la pesquería de tilapia Oreochromis spp. (Pisces: Cichlidae) en la Presa Adolfo López Mateos, Michoacán-Guerrero. Tesis Doctorado, Universidad Nacional Autónoma de México, México.

Jiménez-Badillo, L. 2006. Age-growth models for tilapia Oreochromis aureus (Perciformes, Cichlidae) of the Infiernillo reservoir, Mexico and reproductive behaviour. Rev. Biol. Trop. 54: 277-588.

Meekan, M.G., J.J. Dodson, S.P. Good \& D.A.J. Ryan. 1998. Otholith and fish size relationship, measurement error, and size-selective mortality during the early life of Atlantic salmon (Salmon salar). Can. J. Fish. Aquat. Sci. 55: 1663-1673.
Morales-Nin, B. 1989. Growth determination of tropical merine fishes by means of otholith interpretation and length frequency analysis. Aqua. Liv. Res. 2: 241-253.

Palacios, S.S. 1995. Estudio biológico pesquero de la Tilapia Oreochromis aureus en la presa Adolfo López Mateos (El Infiernillo), Michoacán-Guerrero, México. Tesis Licenciatura, Universidad Nacional Autónoma de México, México.

Pérez, O.G. \& S. Patlani. 2002. Edad y crecimiento de tilapia (Oreochromis niloticus) de la Presa Emiliano Zapata, Morelos. Tesis de Licenciatura, Universidad Nacional Autónoma de México, Zaragoza, México.

Schneider, J.C., P.W. Laarman \& H. Gowing. 2000. Age and growth methods and state averages. In J.C Schneider (ed.). 2000. Manual of fisheries survey methods II: with periodic updates. Michigan Department of Natural Resources, Canada.

Sirios, P., F. Lecomte \& J.J. Dodson. 1998. An otolith-based back-calculation method to account for time-varying growth rate in rainbow smelt (Osmerus mordax) larvae. Can. J. Fish. Aquat. Sci. 55: 2662-2671.

Pauly, D. 1983. Algunos métodos simples para la evaluación de recursos pesqueros tropicales. FAO. Fisheries Technical Paper, 234. FAO, Roma, Italia.

Sparre, P. \& S.C. Venema. 1997. Introducción a la evaluación de recursos pesqueros tropicales. Parte I. Manual. Documento Técnico de Pesca, No. 306.1 Rev. 2. FAO, Chile.

Trewavas, E. 1983. Tilapiine fishes of the genera Sarotherodon, Oreochromis and Danakilia. British Mus. Nat. Hist., Londres, Inglaterra.

Von Bertalanffy, L. 1938. A quantitative theory of organic growth (Inquires on growth Iasw II) Hum. Biol. 10:181-213

Weatherley, A.H. \& H.S. Gill. 1987. The biology of fish growth. Academic, Londres, Inglaterra.

Zar, H.J. 1974. Biostatistical Analysis. Prentice-Hall, Nueva Jersey, EEUU. 